\title{
Optimized cDICE for Efficient Reconstitution of Biological Systems in Giant Unilamellar Vesicles
}

\author{
Lori Van de Cauter, ${ }^{\S}$ Federico Fanalista, ${ }^{\S}$ Lennard van Buren, ${ }^{\S}$ Nicola De Franceschi, Elisa Godino, \\ Sharon Bouw, Christophe Danelon, Cees Dekker, Gijsje H. Koenderink,** and Kristina A. Ganzinger*
}

Cite This: ACS Synth. Biol. 2021, 10, 1690-1702

Read Online

\section{ACCESS | Lill Metrics \& More | 国 Article Recommendations ｜（） Supporting Information}

ABSTRACT: Giant unilamellar vesicles (GUVs) are often used to mimic biological membranes in reconstitution experiments. They are also widely used in research on synthetic cells, as they provide a mechanically responsive reaction compartment that allows for controlled exchange of reactants with the environment. However, while many methods exist to encapsulate functional biomolecules in GUVs, there is no one-size-fits-all solution and reliable GUV fabrication still remains a major experimental hurdle in the field. Here, we show that defect-free GUVs containing complex biochemical systems can be generated by optimizing a doubleemulsion method for GUV formation called continuous droplet interface crossing encapsulation (cDICE). By tightly controlling
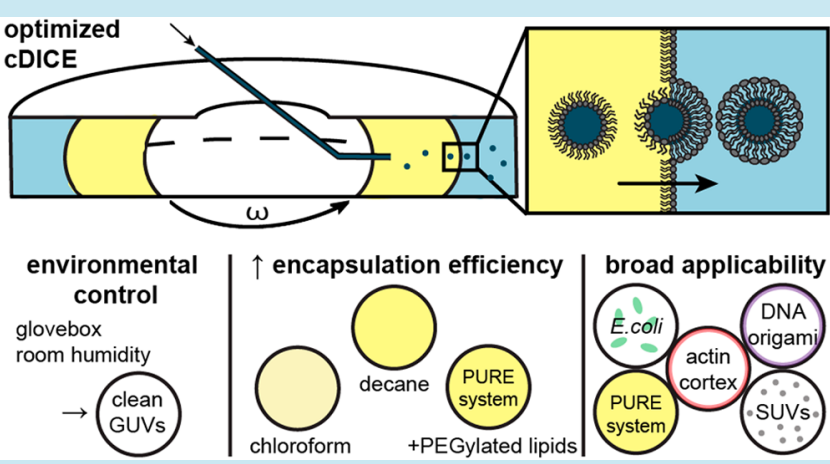
environmental conditions and tuning the lipid-in-oil dispersion, we show that it is possible to significantly improve the reproducibility of high-quality GUV formation as well as the encapsulation efficiency. We demonstrate efficient encapsulation for a range of biological systems including a minimal actin cytoskeleton, membrane-anchored DNA nanostructures, and a functional PURE (protein synthesis using recombinant elements) system. Our optimized cDICE method displays promising potential to become a standard method in biophysics and bottom-up synthetic biology. KEYWORDS: bottom-up synthetic biology, GUVs, emulsion transfer, synthetic cell, actin cytoskeleton, in vitro transcription-translation

$\mathrm{C}$ ellular life is enabled by countless interacting molecules and biochemical reactions with a high degree of interconnectivity and redundancy. Reconstituting cell biological processes using only their minimal functional units from the bottom-up is therefore very helpful to study cellular mechanisms on a molecular and mechanistic level. ${ }^{1-3}$ The field of bottom-up synthetic biology has gained a lot of traction over the past decade, an evolution synchronized with the emergence of several different consortia worldwide to lead the journey toward functional reconstitution of all basic cellular functions, culminating in the creation of a minimal synthetic cell. ${ }^{4-7}$

In this synthetic cell community, giant unilamellar vesicles (GUVs) are widely used as cell-sized, lipid bilayer-enclosed reaction compartments that can be visualized by real-time microscopy and directly manipulated using biophysical tools. ${ }^{8-11}$ Using GUVs as a basis for a functional synthetic cell requires encapsulation of different biological modules in a precise stoichiometry, consisting of a variety of biomolecules ranging in size and charge. However, state-of-the-art GUV fabrication methods are still far from ideal in establishing complex reconstituted systems. On the one hand, easy-toimplement and high-yield methods, such as natural swelling, ${ }^{12}$ electroformation, ${ }^{13-16}$ and gel-assisted swelling, ${ }^{17-20}$ offer poor control over encapsulation efficiency and stoichiometry, and inconveniently contain the same solution on the in- and outside. On the other hand, emulsion-based techniques, in which GUVs are generated from water-in-oil droplets crossing an oil-water interface (using gravity, centrifugation, microfluidic devices, or microfluidic jetting ${ }^{21-27}$ ), offer more control over GUV content and size monodispersity, but at the cost of being less reliable and more technologically advanced, and therefore less accessible.

A promising method that is increasingly being used for complex reconstitutions is continuous droplet interface crossing encapsulation (cDICE). This double-emulsion based technique relies on the continuous transfer of capillarygenerated water-in-oil droplets across an oil-water interface using centrifugal force. ${ }^{28}$ Requiring only easy-to-operate laboratory instrumentation, cDICE can in principle provide high yields while being less technologically demanding than microfluidic-based approaches and allowing for more control

Received: February 24, 2021

Published: June 29, 2021 
a

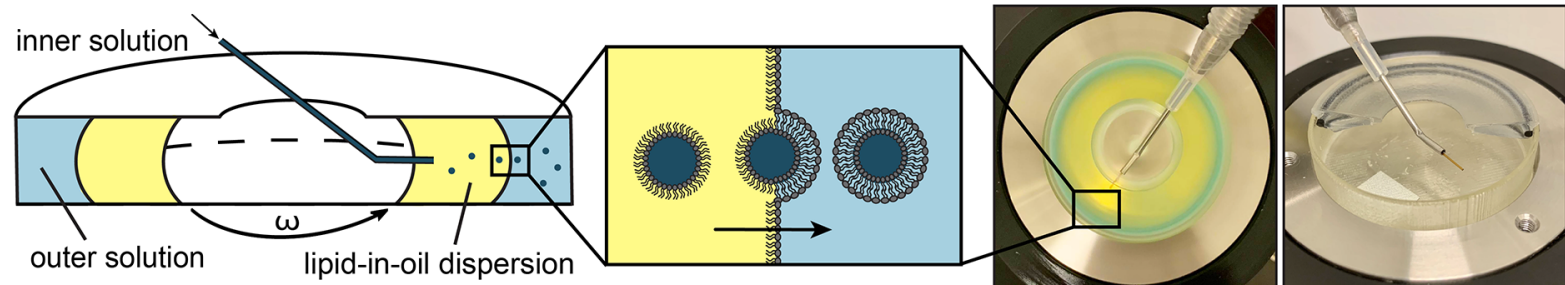

b

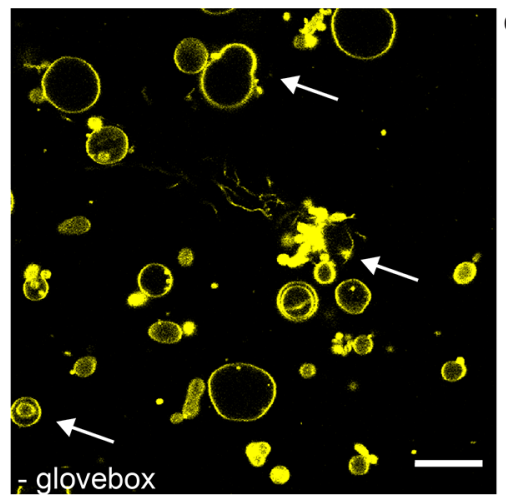

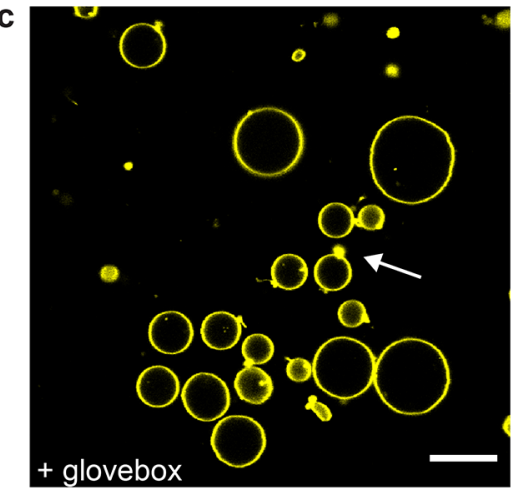

d

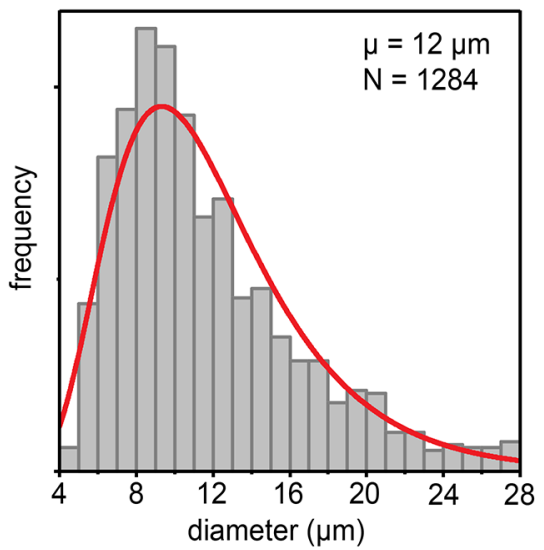

Figure 1. General overview of the cDICE technique and influence of environmental conditions. (a) Cross-sectional schematic of the cDICE method. The center image displays the $3 \mathrm{D}$ printed rotation chamber, with the different fluid layers colored differently for illustration purposes. The rightmost image displays the custom-built spinning device that accommodates the 3D printed rotation chamber. The capillary is inserted using an adjustable magnetic base to allow spatial flexibility upon insertion. During experiments, this setup is connected to a syringe and syringe pump. (b) Representative field of view of GUVs formed using a chloroform-based lipid-in-oil dispersion prepared outside of the glovebox. ATTO 655 DOPE was used as a membrane stain and images were taken using confocal microscopy. Most GUVs contain artifacts in the lipid membrane, and examples are indicated with arrows. Scale bar indicates $20 \mu \mathrm{m}$. (c) Representative field of view of GUVs formed using the final protocol including the use of a glovebox. ATTO 655 DOPE was used as a membrane stain and images were taken using confocal microscopy. Most GUVs are spherical and possess a clean membrane, and only a small population of GUVs still shows artifacts, as indicated with an arrow. Scale bar indicates $20 \mu \mathrm{m}$. (d) Size distribution of GUVs made of DOPC lipids, obtained by the optimized protocol. The distribution is fitted to a log-normal function (red curve).

over size and encapsulated content than swelling methods. ${ }^{28,29}$ However, despite promising first outcomes, using cDICE for protein encapsulation has remained difficult, beyond a few specific cases. ${ }^{30-33}$ At least in part, this is likely due to our lack in understanding of the physical process of vesicle formation and of which parameters are essential to control tightly for the method to work robustly. Significant lab-to-lab variability and constant adaptations to the protocol devised by various laboratories ${ }^{28-30,33}$ have also made it hard to reproduce results across different institutions, leading to the technique being far from accessible.

Here, we aimed to gain a better understanding of the parameters influencing both vesicle formation and encapsulation efficiency in CDICE, allowing us to design an accessible, robust, and reproducible workflow for different encapsulation needs. We show that control of environmental conditions is crucial for reliable formation of defect-free GUVs (i.e., the vesicular membrane is uniform at optical length-scales and does not contain visible lipid pockets) at high yields. Furthermore, we demonstrate different approaches for enhancing the encapsulation efficiency of cDICE by changing the composition of the lipid-in-oil dispersion. We thus provide future users with a detailed protocol for GUV fabrication and a toolbox that can form a firm basis for further experimentspecific optimization. By reproducing key experiments across multiple laboratories in different locations and encapsulating a large variety of biological systems, from the encapsulation of purified proteins to the PURE in vitro transcription-translation system, membrane-anchored DNA origami, and bacteria, we show robustness and versatility of the method. Overall, we demonstrate that our improved cDICE protocol shows great promise for a wide range of complex reconstitution processes in the future, overcoming a major hurdle on the route toward functional synthetic cells.

\section{RESULTS}

Environmental Control Is Essential for Producing Defect-Free GUVs with CDICE. To improve the robustness of the cDICE method, we sought to systematically screen various experimental parameters that might influence GUV formation in cDICE. A typical cDICE setup (Figure 1a) consists of a rotating chamber containing two concentric fluid layers: an inner, lower-density lipid-containing oil phase and an outer, aqueous layer. The aqueous solution to be encapsulated is injected into the lipid-in-oil layer through a capillary, leading to the formation of water-in-oil droplets at the capillary orifice. As these droplets travel outward and traverse the interface of the oil with the outer aqueous phase, a bilayer is formed, yielding GUVs, collected in the outer layer of the system (Figure 1a). GUV formation is thus dependent on the properties of all phases and on other experimental parameters, such as rotation speed and capillary size. ${ }^{28}$ When we sought to enhance the consistency of vesicle production in this inherently sensitive experimental system, the first striking improvement was made by using a chloroform-based lipid-inoil dispersion $^{33}$ as oil phase and preparing it in a humidity-free environment, i.e., inside a glovebox. Without the use of a glovebox, GUVs were generated but the sample contained a lot 


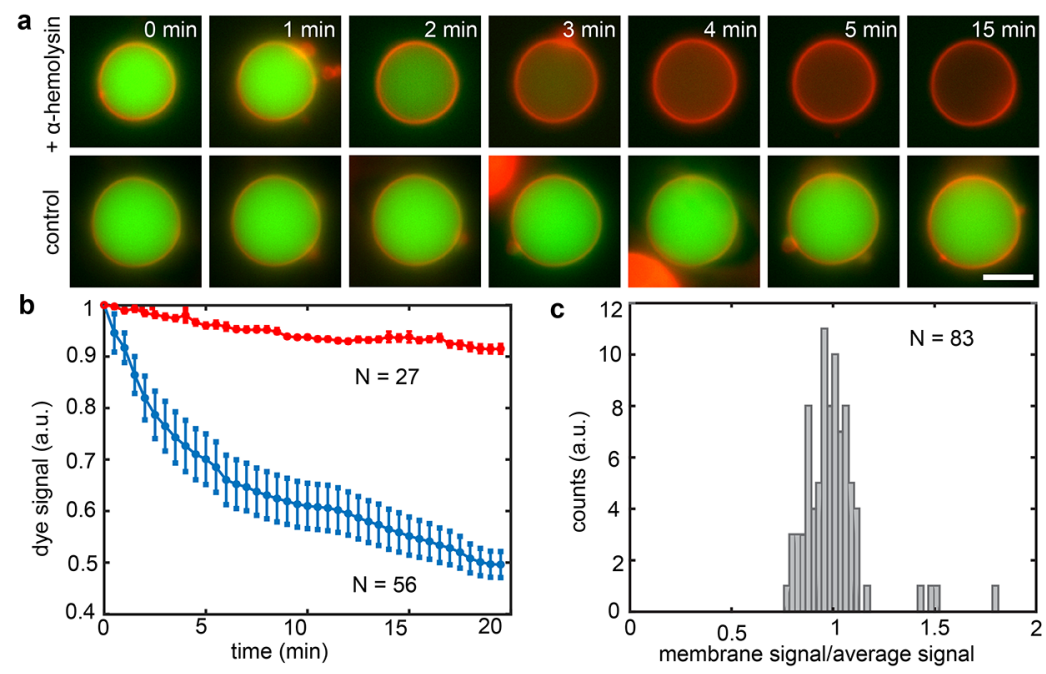

Figure 2. Incorporation of alpha-hemolysin pore protein demonstrates unilamellarity of GUV membrane. (a) Fluorescence microscopy images of single GUVs prepared using a chloroform-based lipid-in-oil dispersion showing different membrane permeability in presence (top row) or absence (bottom row) of alpha-hemolysin. When the pore protein is added to the lipid membrane (red, rhodamine-PE membrane stain), the encapsulated fluorescent dye (green, Alexa Fluor 488) is released in the outer environment within a few minutes. When only alpha-hemolysin buffer is added as a control instead, fluorescent molecules are retained within the GUV volume. Scale bar indicates $5 \mu \mathrm{m}$. (b) Quantitative analysis of GUV fluorescent content loss over time. In presence of alpha-hemolysin (blue curve), Alexa Fluor 488 signal intensity decreases down to $50 \%$ of the initial value within the first $20 \mathrm{~min}$, while in absence of pores (red curve) only a minor decrease $(<10 \%)$, likely due to photobleaching, is detected. (c) Histogram showing GUV membrane fluorescence intensities compared to the overall GUV population.

of residual membrane material, such as free tubes and fluorescent aggregates, and the vast majority of GUVs showed visible fluorescent pockets or budding membrane structures (Figure 1b). In contrast, when the lipid-in-oil dispersion was prepared in a glovebox, samples were much cleaner with most GUVs having quasi-spherical shapes without visible lipid pockets or budding membrane structures (Figure 1c).

In line with this observation, preparation of the lipid-in-oil dispersion inside a glovebox also affected its macroscopic appearance: oil dispersions prepared in a humidity-free environment were transparent, while preparations outside a glovebox yielded visibly opaque dispersions, as quantified by turbidity measurements $\left(A_{350}=0.10 \pm 0.05\right.$ vs $0.42 \pm 0.10$, Figure S1). Furthermore, we analyzed the lipid adsorption kinetics of the different oil dispersions using pendant drop measurements, ${ }^{34}$ where a drop of aqueous solution is suspended in a lipid-in-oil mixture, mimicking the process happening at the orifice of the cDICE capillary. Without humidity control, interfacial tension decreased much faster (Figure S2), indicating faster adsorption of lipids to the oilwater interface. In combination with the adverse effect on vesicle quality, our experiments suggest that presence of water in the lipid-in-oil dispersion interferes with vesicle formation and bilayer quality via changing the microscopic organization of the lipids and their adsorptive behavior.

It is well-known that humidity values change throughout the year, reaching highest values in summer. This seasonal dependency in daily relative humidity can be as large as several tens in percentage, ${ }^{35}$ equivalent to the range of $40-$ $75 \%$ that we observed in the lab. Given the importance of humidity in preparation of the lipid-in-oil dispersion, we extended environmental control to regulating humidity in the room where the cDICE experiments were performed by using a dehumidifier. Indeed, dehumidification down to $30-40 \%$ resulted in smaller variability between lipid adsorption kinetics as measured in pendant drop experiments (Figure S2), indicating a more reproducible adsorption behavior. In line with the lower variability found in lipid adsorption rates, dehumidification also proved to be essential for reliable production of clean vesicles throughout the year. Taken together, using a glovebox for preparation of the lipid-in-oil dispersion and storage of its components, and performing cDICE experiments in a continuously dehumidified room, resulted in a robust formation of clean GUVs.

In the original cDICE paper, ${ }^{28}$ as well as in other follow-up studies, ${ }^{29,30,36,37}$ injection capillaries were pulled from glass tubes to final orifice diameters of a maximum of $20 \mu \mathrm{m}$. Since we found these narrow glass capillaries to be a significant source of experimental variation and problems due to easy clogging of the orifice, we instead used commercially available fused silica capillary tubing with larger diameters $(25,50$, and $100 \mu \mathrm{m})$ to allow for more consistent results, as previously used by Litschel et al. ${ }^{33}$ We found that using all three capillary sizes, our chloroform-based lipid-in-oil dispersion and optimized workflow led to high yields of GUVs with a mean diameter of $12 \mu \mathrm{m}$ and coefficient of variation of $47 \%$ for a capillary size of $100 \mu \mathrm{m}$ and rotation speed of $1900 \mathrm{rpm}$ (Figure 1d). The size distributions of the GUVs did not significantly change across the different capillary sizes (Figure S3) and they were broader than the ones previously obtained for smaller orifice sizes. ${ }^{28}$ However, the lack of control over GUV size is compensated by a much-improved reliability of encapsulation and GUV formation due to avoidance of clogging, in particular for $100 \mu \mathrm{m}$ fused silica capillaries. Other capillary materials were also successfully used, i.e., 100 $\mu \mathrm{m}$ PEEK capillary tubing. Changes in rotation speed (1000$2900 \mathrm{rpm}$ ) also did not alter the size distributions for the different orifice diameters (Figure S3). No precise control of rotation speed is thus needed in order to get robust GUV formation, with size distributions in an ideal range for bottomup reconstitution of eukaryotic cells. In terms of yield, the absolute number of GUVs obtained using the optimized 

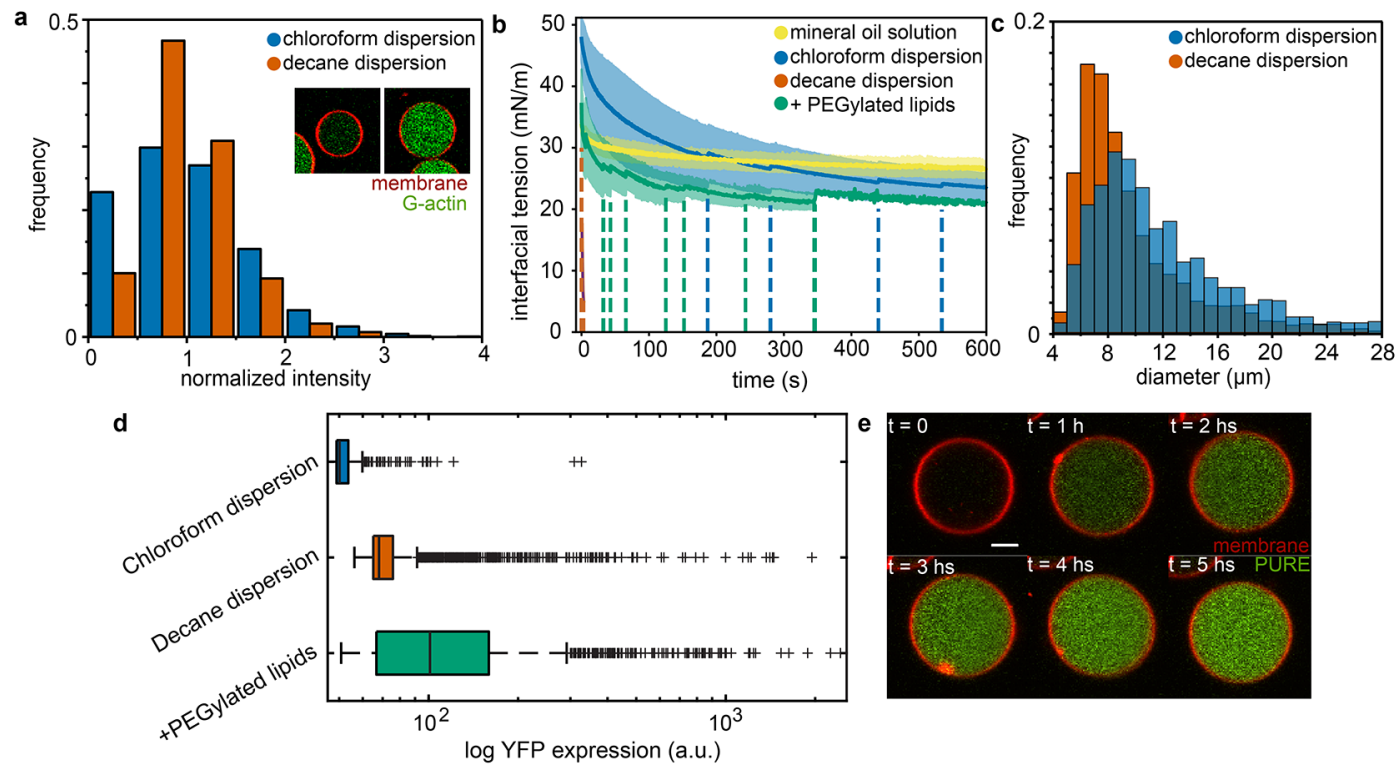

$\log$ YFP expression (a.u.)

Figure 3. Improved encapsulation by tuning of the lipid-in-oil dispersion. (a) Encapsulation efficiency of G-actin using a chloroform-based lipid dispersion (blue) and decane-based lipid dispersion (orange). The first bin represents GUVs with very low fluorescence intensity, and represents $23 \%$ of the population for the chloroform-based lipid dispersion and only $10 \%$ for the decane-based lipid dispersion. (b) Interfacial tension decrease measured for a pendant droplet of G-buffer in different lipid-in-oil mixtures. Solid lines show averaged data with standard deviation for a lipidchloroform solution in mineral oil only (yellow, $n=9$ ), dispersed lipid aggregates using chloroform (blue, $n=13$ ), or decane (orange, $n=7$ ) in silicone oil:mineral oil 80:20 and a chloroform-based lipid-in-oil dispersion with $0.01 \mathrm{~mol} \%$ of PEGylated lipids (green, $n=9)$. The dashed lines indicate individual events where the droplet fell off, which gave rise to apparent jumps in the averaged curves. When using the decane-based dispersion, all droplets detached within seconds. (c) Size distribution of GUVs made using a chloroform-based lipid dispersion (blue) and decanebased lipid dispersion (orange). (d) Box plots of the YFP expression after $5 \mathrm{~h}$ of incubation in GUVs obtained using dispersed lipid aggregates using chloroform (blue), decane (orange), and a chloroform-based lipid-in-oil dispersion with $0.01 \mathrm{~mol} \%$ of PEGylated lipids (green). The boxes represent IQR (25th-75th percentiles), the center line indicates the median and the whiskers extend to the maximum and minimum value excluding outliers. Outliers are individually indicated using plus symbols. (e) Time-lapse images of YFP expression in a single GUV using a chloroform-based lipid-in-oil dispersion with $0.01 \mathrm{~mol} \%$ of PEGylated lipids. Scale bar indicates $5 \mu \mathrm{m}$.

cDICE protocol is dependent on total encapsulation volume, flow rate, and characteristics of the used biological agents. From the average number of GUVs visible per field of view, we estimate the absolute number of GUVs to reach well over 1000 vesicles in a typical experiment $(100 \mu \mathrm{L}$ of inner aqueous solution and a flow rate of $\left.25 \mu \mathrm{L} \mathrm{min}{ }^{-1}\right)$.

Unilamellarity of cDICE-Produced GUVs. Many reconstitution experiments require unilamellar lipid membranes, as this determines permeability and mechanical properties of the GUV and is needed for insertion of transmembrane proteins, including pore proteins, into the bilayer. Therefore, we next aimed to investigate if our GUV membranes were unilamellar by monitoring insertion of alpha-hemolysin, a protein that assembles a heptameric pore structure in the lipid membranes with a diameter of $14 \AA$, through which small molecules can pass and which is highly sensitive to the thickness of lipid bilayers. $^{38,39}$ As a tracer, we encapsulated $5 \mu \mathrm{M}$ of the fluorescent dye Alexa Fluor 488 (643 Da) and we immobilized the GUVs within a polyisocyanide hydrogel ${ }^{40}$ to aid long-term imaging. ${ }^{41}$ After that, alpha-hemolysin was added to the chamber and fluorescent imaging was immediately started. Within minutes following alpha-hemolysin addition, all GUVs observed started to lose their fluorescent content and all had lost $50 \%$ of their content after $\sim 20 \mathrm{~min}$ (Figure 2a, top row; Figure $2 \mathrm{~b}$, red curve). In stark contrast, when only alphahemolysin buffer was added to the GUVs as a control, fluorescent molecules were clearly retained within all GUVs (Figure 2a, bottom row; Figure 2b, blue curve). This indicated that loss of GUV content was due to pore formation and hence membrane unilamellarity. Furthermore, individual GUV membrane intensities normalized by the population's mean membrane intensity are consistently distributed around unity, indicating a homogeneous lamellarity over the GUV population (Figure 2c). Taken together, our results clearly show that the cDICE method produces unilamellar GUVs.

Improvement of Encapsulation Efficiency. To allow for complex reconstitution experiments, it is essential to have control over the encapsulation of functional biomolecules in the right stoichiometric ratios. We probed the encapsulation efficiency of our improved cDICE protocol by encapsulation of the cytoskeletal protein actin, a broadly used protein in the synthetic biology field. ${ }^{42}$ While all experiments using our optimized cDICE protocol resulted in successful encapsulation of monomeric actin in GUVs at high vesicle yields, automated analysis of actin fluorescence at the equatorial plane of the GUV from confocal fluorescence imaging surprisingly revealed a substantial fraction of GUVs with very low actin content, indicating that many of the formed vesicles were seemingly empty (23\%, Figure 3a, Figure S4a). We tested if the encapsulation efficiency could be improved by using different lipid-in-oil mixtures. We reasoned that the encapsulation efficiency may depend on the lipid adsorption kinetics, as it has been reported earlier that the dispersion method of lipids had a strong effect on their adsorptive behavior. ${ }^{43}$ Therefore, we investigated the effect of lipid dispersion strategy on adsorption kinetics, GUV formation, and encapsulation efficiency for three lipid mixtures: lipids in chloroform dispersed as aggregates in a 80:20 mixture of silicon and mineral oil as mentioned above, a 


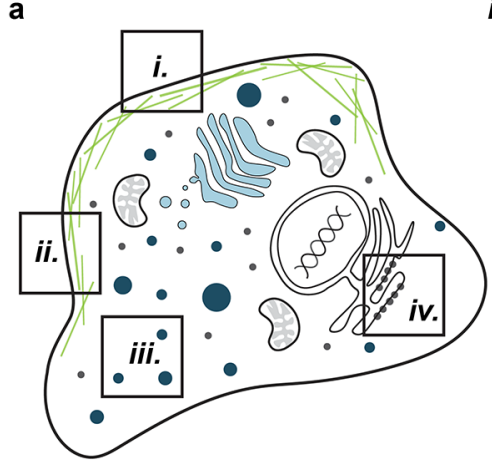

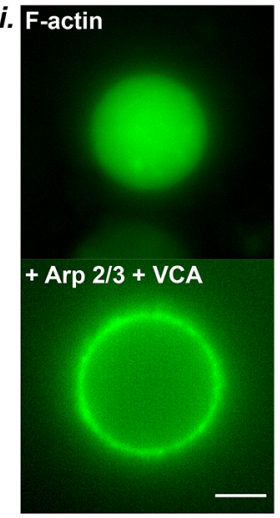
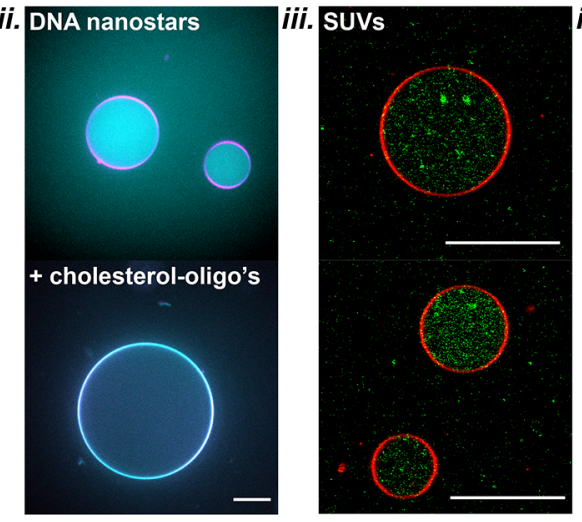

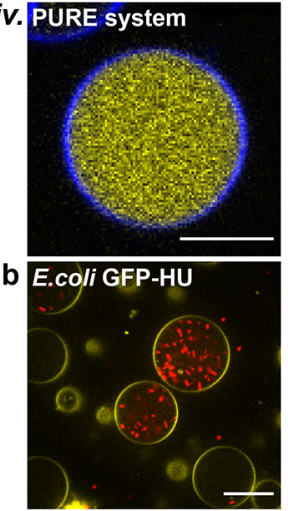

Figure 4. Proof-of-concept experiments showing versatility of cDICE and its applicability for the synthetic cell community. (a) Overview: GUVs as artificial membrane systems to mimic cellular membranes and membrane interactions. ( $i$ ) Reconstitution of a minimal actin cortex inside a GUV, nucleated at the vesicular membrane by the Arp $2 / 3$ complex, the C-terminal VCA domain of WASp, and profilin. Scale bar indicates $5 \mu$ m. (ii) Encapsulation of DNA origami nanostructures, freely diffusing inside the GUV lumen and capable of membrane localization upon addition of $2 \mu \mathrm{M}$ of cholesterol-oligonucleotides. Scale bar indicates $15 \mu \mathrm{m}$. (iii) Encapsulation of SUVs inside GUVs to form a multicompartmentalized system. Scale bars indicate $20 \mu \mathrm{m}$. (iv) Encapsulation of PUREfrex2.0 and DNA encoding for YFP. Scale bar indicates $10 \mu \mathrm{m}$. (b) Encapsulation of GFPHU expressing E. coli bacteria. A large number of bacteria could be observed inside the GUV lumen, clearly viable as evident from their motility. Scale bar indicates $20 \mu \mathrm{m}$.

similar dispersion of lipid aggregates but using decane instead of chloroform, and a lipid-chloroform solution in mineral oil only. Chloroform and decane serve as good solvents for the lipids, while the lipids do not dissolve in the oils. This way, we aimed to produce different lipid-in-oil dispersions with various aggregation states, with the mineral oil dispersion having smallest aggregate size, and both chloroform- and decanebased lipid dispersions having larger aggregate sizes. ${ }^{43}$

First, we confirmed the aggregation state of the lipids by absorbance measurements. Indeed, the mineral oil dispersion was much less turbid $\left(A_{350}=0.03 \pm 0.01\right)$ than the chloroform- or decane-based dispersion $\left(A_{350}=0.10 \pm 0.05\right.$ and $A_{350}=0.20 \pm 0.12$ respectively, Figure $\left.S 1\right)$, indicating that the latter two have a higher propensity to form aggregates. Pendant drop measurements showed that dispersing lipids as aggregates using chloroform resulted in fast lipid adsorption (Figure $3 b$, blue curve), indicating fast monolayer formation. The decane-based lipid dispersion resulted in even faster adsorption, with all droplets detaching within several seconds (Figure $3 b$, orange curve). In contrast, lipids dispersed in mineral oil exhibited a slower and smaller decrease of interfacial tension (Figure $3 \mathrm{~b}$, yellow curve), meaning slow adsorption of lipids to the oil-water interface and a small coverage of the final interface. In line with the idea that faster stabilization of the oil-water interface by faster lipid adsorption leads to more robust monolayer formation, we observed no GUV formation when using lipids dispersed in mineral oil, whereas experiments using lipids dispersed as aggregates in a 80:20 mixture of silicon and mineral oil using chloroform or decane gave large GUV yields (Figure S4).

We then tested if the fast-adsorbing decane mixture could improve the encapsulation efficiency of cDICE. In stark contrast to the encapsulation of G-actin using chloroform as an organic solvent, using a decane-based lipid dispersion resulted in a significant decrease of the fraction of seemingly empty vesicles ( $10 \%$ vs $23 \%$, Figure 3a, Figure S4). Although large differences in both adsorption kinetics and encapsulation efficiency can be observed between decane- and chloroformbased lipid-in-oil dispersions, they yield GUVs similar in size distribution, size polydispersity, and visual membrane cleanli- ness (Figure 3c, Figure S4). We also note that the lipid adsorption behavior of the chloroform-based dispersion is highly variable, much more so than for decane-based lipid dispersions or lipids dispersed in mineral oil only (Figure $3 \mathrm{~b}$ ). Since the lipid dispersions are metastable mixtures and chloroform readily evaporates under ambient conditions, changes to their composition happen on time scales similar to the experimental runtime. Indeed, time-dependent absorbance measurements indicated a rapid change in oil turbidity, indicative of an increase in aggregate size, on the time scale of minutes, confirming the intrinsic instability of chloroformbased lipid dispersions (Figure S5).

Efficient encapsulation is particularly important for reconstitution of cell-free gene expression reactions (in vitro transcription-translation systems) within GUVs, as the relative stoichiometry of their components has to be rather closely retained for optimal functioning. ${ }^{44}$ Functionality might further be affected by possible hydrophobic interactions of the protein components with organic solvents during encapsulation, although some groups already successfully encapsulated in vitro transcription-translation systems with emulsion-droplet transfer- $^{45-47}$ and microfluidic-based methods. ${ }^{48}$ To our knowledge, functional encapsulation of a cell-free gene expression (e.g., the Protein synthesis Using Recombinant Elements (PURE) system ${ }^{49}$ ) has never been demonstrated for GUVs produced with the cDICE method. We therefore explored if we could encapsulate the PURE system using our improved cDICE protocol. To this end, GUVs encapsulating PUREfrex2.0, a commercially available PURE system, along with a linear DNA construct coding for yellow fluorescent protein (YFP), were produced using both a chloroform-based lipid dispersion and a decane-based lipid dispersion. Gene expression in GUVs incubated at $37^{\circ} \mathrm{C}$ was monitored by imaging YFP production within the GUV lumen over time. We observed that the different dispersion strategies used for GUV fabrication influenced the level of gene expression: the distribution of luminal fluorescence intensity after $5 \mathrm{~h}$ of gene expression employing decane-based lipid aggregates showed improved gene expression levels compared to the encapsulation using chloroform-based lipid aggregates, which 
barely yielded any YFP expressing GUVs at all. Nevertheless, both gene expression levels and numbers of YFP expressing GUVs were still very low (Figure 3d and Figure S6a,b).

In addition to the lipid dispersion strategy, the lipid composition of the bilayer membrane can also alter adsorption kinetics and hence improve encapsulation efficiency. In particular, PEGylated lipids, lipids with a flexible poly(ethylene) glycol (PEG) linker, are often proposed to boost robust vesicle formation for various protocols. ${ }^{17,50-52} \mathrm{We}$ therefore investigated if doping the vesicular membrane with $0.01 \mathrm{~mol} \%$ 18:0 PEG2000 PE could improve encapsulation of the PURE system when using cDICE. The presence of PEGylated lipids slightly increased the adsorption rate of lipids to the oil-water interface (Figure $3 b$, green curve). Interestingly, doping the membrane with $0.01 \mathrm{~mol} \%$ PEGylated lipids greatly enhanced expression of the encapsulated PURE system and resulted in the highest gene expression levels and a large population of GUVs expressing YFP (Figure 3d, e and Figure S6c). These results show that optimization of encapsulation efficiency both via lipid dispersion and lipid composition is crucial to allow for functional reconstitution of complex reactions such as the PURE system in GUVs made using cDICE.

Proof-of-Concept Experiments Illustrate Versatility of the Optimized Workflow. Finally, to investigate the broad applicability of our improved cDICE method, we aimed to reconstitute a wide range of minimal systems inside cDICEmade GUVs (Figure 4). First, we encapsulated a minimal, branched actin network. In eukaryotic cells, the actin cortex is the protein machinery responsible for cell division. ${ }^{53,54}$ Reconstitution of a functional actin cortex anchored to the inner leaflet of the GUV membrane therefore offers an attractive route to induce GUV constriction, and possibly membrane fission, in synthetic cells. Our minimal actin cortex consisted of actin together with the verpolin homology, cofilin, and acidic domain of the Wiscot-Aldrin Syndrome protein (VCA), the Arp $2 / 3$ complex, and profilin. The Arp $2 / 3$ complex is an actin nucleator responsible for promoting formation of a branched actin network at the cell membrane. ${ }^{55,56}$ VCA was His-tagged to be able to bind to DGS-NTA(Ni) lipids in the membrane. ${ }^{57,58}$ Together with Arp2/3, VCA promoted localized nucleation of a branched cortex at the membrane, while profilin was used to prevent actin polymerization in the GUV lumen. ${ }^{59,60}$ Actin displayed a clear localization at the GUV membrane (Figure 4a, $i$, Figure S7a), similarly to what was obtained using other GUV fabrication methods. ${ }^{61,62}$ In the absence of membrane anchors and nucleators, actin was uniformly distributed within the GUV volume (Figure 4a,i).

As a synthetic mimic of the cellular actin cortex, we encapsulated DNA origami nanostructures ${ }^{63}$ that are capable of lateral cross-linking at the vesicular membrane. These fourarmed DNA assemblies (Figure S8) diffuse freely in the lumen of the GUV but were efficiently recruited to the membrane upon co-encapsulation of a cholesterol-oligonucleotide membrane anchor that binds single-stranded DNA sites on the origami (Figure 4a, ii, Figure S7b). Here, the monomeric DNA tiles freely diffuse in the membrane plane and form a precortex. We also successfully encapsulated small unilamellar vesicles (SUVs, $\sim 100 \mathrm{~nm}$ diameter), ${ }^{9}$ mimicking multicompartmental cellular systems (Figure 4a, iii, Figure S7c). In the future, these compartments could be designed to trigger or sustain intravesicular reactions, allowing control over biochemical reactions inside the GUV lumen. ${ }^{64-66}$

Furthermore, as mentioned above, our cDICE method can be used to encapsulate a functional in vitro transcriptiontranslation system (the PURE system), provided PEGylated lipids are included in the lipid mixture (Figure $4 a, i v)$. The broad applicability of cDICE is further demonstrated by the successful encapsulation of objects that are large compared to the GUV size, i.e., entire E. coli bacteria (Figure 4b, Figure S7d). Cylindrical in shape, with a length of approximately 3 $\mu \mathrm{m}$ and a diameter of $1 \mu \mathrm{m},{ }^{67}$ these are several orders of magnitude larger than even many DNA origami structures. The bacteria were clearly mobile inside the GUVs (Movie S1), showing that the cDICE process does not significantly affect their viability. Encapsulating live bacteria inside synthetic cells could be a promising route to combine "the best of both worlds", e.g., photosynthetic cyanobacteria could be repurposed as "chloroplasts" for the synthetic cell, similar to a recent study which included chloroplasts isolated from plant cells. ${ }^{68}$

Overall, the improved cDICE method is shown to be capable of encapsulating a variety of functional minimal systems related to cell mechanics, cell metabolism, and gene expression, all required for the generation of a synthetic cell.

\section{DISCUSSION}

A good understanding of the parameters influencing the GUV formation process in CDICE is crucial, especially for design of reconstitution experiments beyond first proof-of-concept experiments. Here, we showed that tight control over the lipid-in-oil mixture is key to successful and reproducible GUV formation. We found that membrane quality, which affects mechanical measurements and quantitative fluorescence analysis, was strongly improved by environmental control over preparation and handling of the lipid-in-oil dispersion, notably handling the lipid dispersion in a humidity-free environment (i.e., a glovebox) and decreasing humidity to $30 \%$ during vesicle formation. We hypothesize that air humidity affects bilayer formation by changing the microscopic aggregation state of the lipid-in-oil mixture, and thereby the lipid adsorption behavior. Partial hydration of lipids could possibly lead to the formation of larger lipid aggregates, such as reverse micelles or lamellar structures, hindering proper monoand bilayer formation. Yet, fully understanding the microscopic mechanics of this thermodynamically unstable, multicomponent system remains difficult. ${ }^{69,70}$ Importantly, we also demonstrated the unilamellarity of the formed GUVs by correct insertion of alpha-hemolysin to allow pore formation. Although the appearance of the GUV membranes was visibly improved upon environmental control, a common concern remains the possible presence of residual oil traces in the membrane. However, it was shown in previous work that cDICE-formed GUVs are unlikely to have large traces of oil persisting in the membrane. ${ }^{28,29}$ It is unknown whether transmembrane proteins are affected by the presence of residual oil in vesicular membranes but interestingly, recent work indicates that it does not significantly alter the static, mechanical membrane properties of the GUVs compared to electroformed GUVs. ${ }^{71-73}$ Altogether, this makes vesicle formation with the improved protocol compatible with reconstitution experiments requiring clean unilamellar membranes, such as studies involving membrane mechanics or membrane permeability. 
Furthermore, we showed that the dispersion state of the lipids is crucial for efficient GUV formation using cDICE. As other existing protocols show, many different lipid-in-oil mixtures can be used for GUV formation. ${ }^{28-30,33,43}$ In particular, Claudet et $a l^{43}$ found lipids dispersed as aggregates in an oil phase to promote more efficient bilayer formation. We provide experimental evidence that indeed the lipid bulk aggregated state strongly influences adsorption kinetics and thereby vesicle formation, supporting and explaining the observations of Claudet et al. ${ }^{43}$ Our tensiometry findings also indicate that not solely adsorption speed is of importance for proper bilayer formation, but the structure and content of the lipid aggregates is equally important for mono- and bilayer formation. Hence, having lipids dispersed as aggregates alongside humidity control is essential for clean GUV formation. This indicates a nontrivial relation between lipid properties, lipid dispersion state, adsorption kinetics and the final membrane quality. Adsorption speed as measured by pendant drop experiments can therefore not be used as a stand-alone quantity to assess whether a given lipid-in-oil mixture will support GUV formation in cDICE. Future research into the molecular mechanisms of the lipid-in-oil dispersions could involve a systematic characterization of the lipid aggregates species via, for example, dynamic light scattering (DLS) or electron microscopy (EM).

By tuning the lipid-in-oil dispersion with different organic solvents or different types of lipids, the encapsulation efficiency of cDICE could be improved. Faster lipid adsorption when using a decane-based dispersion, as compared to using a chloroform-based dispersion, led to a better G-actin encapsulation. For functional encapsulation of the PURE system on the other hand, the presence of PEGylated lipids proved to be crucial. This cell-free expression system has a complex molecular composition and all the individual components need to be present in order to yield a functional readout. While addition of PEGylated lipids has proven to be very effective for encapsulation of the PURE system with cDICE, it should be noted that PEGylated lipids can have adverse effects on protein functionality and membrane physicochemical behavior, as the polymer chains introduce crowding and steric repulsion of components from the membrane as well as affect the membrane thickness. ${ }^{74}$ In this case, our experiments suggest that depending on the encapsulated species, PEGylated lipids can be avoided and high encapsulation efficiencies can be reached instead by changing the solvent.

Our cDICE protocol robustly yields GUVs with an average diameter of $12 \mu \mathrm{m}$ and coefficient of variation of $47 \%$. This size distribution was robust to changes in rotation speed and capillary diameters from $25-100 \mu \mathrm{m}$. This consistency over differences in these two central parameters implies that the workflow we have adopted lies in the jetting regime. ${ }^{75} \mathrm{~A}$ jet at the capillary orifice is broken up into a polydisperse droplet population due to the Rayleigh instability in combination with the centrifugal force applied in cDICE. ${ }^{75} \mathrm{~A}$ high degree of polydispersity can be advantageous for bulk assays to screen multiple conditions in one single experiment, ${ }^{76,77}$ but undesirable for other applications. As Abkarian et al. ${ }^{28}$ showed, decreasing the capillary diameter to values around $10 \mu \mathrm{m}$ or using an additional inner fluid layer to decrease shear forces are viable strategies to achieve more precise size control. However, using these small orifice sizes poses other problems, including fast clogging of small diameter capillaries, rendering the method much less reliable. Here, we demonstrate that to reproducibly encapsulate viscous solutions containing a high concentration of polymerizing protein, as when encapsulating concentrated actin solutions, it is advantageous to use a larger capillary.

Taken together, we have shown that humidity control is essential for reliable production of clean GUVs with cDICE. Furthermore, we found that the encapsulation of different biological systems can be modulated by tuning the lipid-in-oil dispersion and the membrane composition. As a result, the optimized workflow laid out in this research enables the generation of bespoke GUVs at good yields and with high encapsulation efficiency. We showed that encapsulation was compatible with molecular membrane anchors such as the cholesterol-oligonucleotide anchors used with DNA origami and a minimal actin cortex, while maintaining functionality even for complex systems like the PURE system. This renders a method that is robust and achieves reproducible results across many months and multiple laboratories. By conducting several proof-of-concept experiments, we were able to demonstrate the versatility of the cDICE method: from reconstitution of an actin cortex, to encapsulation of a cellfree expression system, membrane-anchored DNA nanostructures, and entire E. coli bacteria, these experiments open up a portal to generating GUVs with contents of ever-greater complexity. In the future, additional modifications by changing experimental parameters such as capillary size, rotation speed, chamber design, etc. can be made to further extend the possibilities of $\mathrm{CDICE}$ and perform experiment-specific optimization. This way, cDICE displays promising potential to become a standard method for the synthetic biology, biochemistry, and biophysics communities in the future.

\section{METHODS}

Design and Fabrication of the Spinning Device/ Rotational Chambers. The cDICE device was designed and developed in-house at AMOLF. A 15-W Maxon EC32 motor (5 wire version, part number 353399) served as the rotating component of the apparatus, providing a wide range of rotation speeds (from $200 \mathrm{rpm}$ up to $6000 \mathrm{rpm}$ ) and allowing precise speed ramps for controlled speeding up and slowing down of rotation. This is especially important to avoid mixing of the solutions after experiments, which would lead to lipid debris in the outer aqueous solution, and to avoid disruption of the formed GUVs. Translucent, cylindrical chambers were designed and printed in-house (Stratasys Objet260 Connex3; Veroclear printing material). The chambers measure $38 \mathrm{~mm}$ in diameter, have an inner height of $7.4 \mathrm{~mm}$, and include a circular opening of $15 \mathrm{~mm}$ in diameter in the top to allow facile access to the solutions with the capillary. The respective designs for rotation chambers and cDICE device are available on GitHub (https://github.com/GanzingerLab). The other laboratories at TU Delft used similar devices.

General cDICE Experimental Workflow. Synthetic fused silica capillary tubing (TSP 100/050/025 375, Molex) was employed due to its highly smooth inner surface, allowing a controlled flow of inner aqueous solutions. It was cut to a length of several centimeters using the supplied cutting stone and attached to a short piece of flexible microbore tubing (Microbore Tubing, 0.020" $\times 0.060^{\prime \prime}$ OD, Cole-Parmer $\mathrm{GmbH}$ ) using two-component epoxy glue (Bison) or instant glue (Pattex). Using a hollow piece of metal, the capillary tubing was then bent so it could be inserted horizontally into 
the rotational chamber. To inject the solutions, this setup was connected to a $250 \mu \mathrm{L}$ glass syringe (SGE Gas Tight Syringe, luer lock, Sigma-Aldrich) using a shortened needle as connector (Hamilton Needle, Metal hub, needle size 22 ga. blunt tip, Sigma-Aldrich). PEEK capillary tubing (PEEK tubing, $1 / 32^{\prime \prime}$ OD $\times 0.10 \mathrm{~mm}$ ID, BGB Analytik) was used in experiments when explicitly specified. The encapsulation solutions contained $18.5 \% \mathrm{v} / \mathrm{v}$ OptiPrep (density gradient medium with a density of $1.320 \mathrm{~g} \mathrm{~mL}^{-1}$ ) to increase the density. Unless specified otherwise, the outer aqueous phase was a solution of glucose in Milli- $Q$ water (concentration adjusted to reach a $10-20 \mathrm{mOsm}$ higher osmolarity compared to the inner aqueous solution). In a typical experiment, the encapsulation solution was loaded into the syringe setup, rotation was started, $700 \mu \mathrm{L}$ of outer aqueous solutions was inserted into the rotating chamber, followed by $5.5 \mathrm{~mL}$ of the lipid-in-oil dispersion. The capillary was then inserted horizontally in the oil layer, until it was visibly embedded. The solution was injected using a syringe pump (KDS $100 \mathrm{CE}$, $\mathrm{KD}$ Scientific) at a rate of $25 \mu \mathrm{L} \mathrm{min} \mathrm{m}^{-1}$, unless specified otherwise. The system was spun for a predetermined time depending on the encapsulation volume. Rotation speed ranged from 1000 to $2700 \mathrm{rpm}$ and the capillary diameter from $25 \mu \mathrm{m}$ to $100 \mu \mathrm{m}$ depending on the experiment type, with $1900 \mathrm{rpm}$ and $100 \mu \mathrm{m}$ being considered the default values. After every experiment, the chamber was tilted and excess oil was removed. The GUVs were then allowed to sink to the bottom of the rotation chamber for $10 \mathrm{~min}$, after which they were harvested using a cut pipet tip and transferred to an observation chamber. Glass coverslips were passivated using 1 $\mathrm{mg} \mathrm{mL} \mathrm{m}^{-1}$ beta-casein in Milli-Q water. Room humidity was kept around 30-40\% using a dehumidifier (TTK 71 E Dehumidifier, Trotec). The other laboratories used a similar workflow, based on this main protocol.

Preparation of Lipid-in-Oil Dispersions. 1,2-Distearoyl$s n$-glycero-3-phosphoethanolamine- $N$-[methoxy (polyethylene glycol)-2000] (18:0 PEG2000 PE), 1,2-dioleoyl-sn-glycero-3phosphoethanolamine- $N$-(lissamine rhodamine $\mathrm{B}$ sulfonyl (18:1 Liss Rhod PE), 18:1 1,2-dioleoyl-sn-glycero-3-phophocholine (DOPC), 1,2-dioleoyl-sn-glycero-3-[( $\mathrm{N}$-(5-amino-1carboxypentyl)iminodiacetic acid)succinyl] (nickel salt) (DGS-NTA(Ni)), and 1,2-dioleoyl-sn-glycer-3-phosphoethanolamine- $N$-(lissamine rhodamine B sulfonyl) (rhodaminePE) were purchased from Avanti Polar Lipids. ATTO 488 and ATTO 655 labeled 1,2-dioleoyl-sn-glycero-3-phosphoethanolamine (DOPE) were obtained from ATTO-TEC. Stock solutions in chloroform were stored at $-20{ }^{\circ} \mathrm{C}$. The lipids were mixed in the desired molar ratio in a $20 \mathrm{~mL}$ glass screw neck vial (Fisherbrand EPA Screw Neck Vial, Fisher Scientific and Fisherbrand $24 \mathrm{~mm}$ PP Screw Seal, Closed Top, 24-400 Thread, Assembled Septum, Fisher Scientific) to obtain a final concentration of $0.2 \mathrm{mg} \mathrm{mL}^{-1}$. After desiccation using a gentle nitrogen flow, the vial was brought inside a glovebox, where the lipid film was resuspended in $415 \mu \mathrm{L}$ of chloroform (Uvasol, Sigma-Aldrich) or $n$-decane (99+\%, pure, Acros Organics). A mixture of $5.2 \mathrm{~mL}$ silicon oil (viscosity 5 cst $(25$ $\left.{ }^{\circ} \mathrm{C}\right)$, Sigma-Aldrich) and $1.3 \mathrm{~mL}$ mineral oil (BioReagent, Sigma-Aldrich) was then added dropwise to the lipids while vortexing. For the lipid dispersion in mineral oil, $6.5 \mathrm{~mL}$ of mineral oil (BioReagent, Sigma-Aldrich) was used instead. After tightly closing the vial and securing the seal with Parafilm, the lipid-in-oil dispersion was vortexed an additional $2.5 \mathrm{~min}$ and sonicated in a bath sonicator for $15 \mathrm{~min}$ while keeping the bath temperature below $40{ }^{\circ} \mathrm{C}$. The mixtures were used the same day in experiments.

UV-Vis Absorbance Measurements. Turbidity measurements were performed by UV-vis absorbance using a Denovix DS-11 spectrophotometer. Lipid-in-oil dispersions were prepared as described above and used directly for absorbance measurements. For each measurement, a cuvette (UV cuvette ultramicro, BRAND) was filled with $100 \mu \mathrm{L}$ of lipid-in-oil dispersion and the absorbance at $350 \mathrm{~nm}$ was measured thrice. Prior to each measurement, a blank was taken using the corresponding oil or oil mix.

Pendant Drop Measurements. Pendant drop measurements were performed using a DSA 30S drop shape analyzer (Kruss, Germany) and analyzed with the Kruss Advanced software. For each measurement, a lipid-in-oil dispersion containing $100 \%$ DOPC was prepared in an identical manner as for cDICE experiments. Directly after vortexing, the mixture was divided over three glass $1.0 \mathrm{~mm}$ cuvettes (Hellma Analytics). In each cuvette, a $30 \mu \mathrm{L}$ droplet containing Gbuffer ( $5 \mathrm{mM}$ tris(hydroxymethyl)aminomethane hydrochloride (Tris- $\mathrm{HCl}) \mathrm{pH} 7.8$ and $0.1 \mathrm{mM}$ calcium chloride $\left(\mathrm{CaCl}_{2}\right)$ ) and $18.5 \% \mathrm{v} / \mathrm{v}$ OptiPrep was formed with a rate of $5 \mu \mathrm{L} \mathrm{s}^{-1}$ using an automated dosing system from a hanging glass syringe with needle diameter of $1.060 \mathrm{~mm}$ (Hamilton). Immediately when the droplet reached its final volume, 100 frames of the droplets shape were first acquired at a frame rate of 5 frames per second after which another 500 frames were taken with 1 frame per second. The droplet contour was automatically detected and fitted with the Young-Laplace equation to yield the interfacial tension. For measurements in dehumidified conditions, a dehumidifier was switched on at least $1 \mathrm{~h}$ prior to the measurement. The lipid-in-oil dispersion was continuously mixed during each measurement using a magnetic stirrer. In several experiments, interfacial tension decreased very rapidly causing the droplet to detach before the end of the measurement.

Alpha-Hemolysin. DOPC (97.4 mol \%), DGS-NTA(Ni) $(2.5 \mathrm{~mol} \%)$, and rhodamine-PE lipids $(0.1 \mathrm{~mol} \%)$ were used for preparation of the lipid-in-oil dispersion as described earlier. GUVs encapsulating F-buffer $(20 \mathrm{mM}$ Tris- $\mathrm{HCl} \mathrm{pH}$ 7.4, $50 \mathrm{mM}$ potassium chloride $(\mathrm{KCl}), 2 \mathrm{mM}$ magnesium chloride $\left(\mathrm{MgCl}_{2}\right), 0.5 \mathrm{mM}$ adenosine triphosphate (ATP) and $1 \mathrm{mM}$ dithiothreitol (DTT)), $18.5 \% \mathrm{v} / \mathrm{v}$ OptiPrep, and $5 \mu \mathrm{M}$ Alexa Fluor 488 (Thermo Fischer) were produced in a 200 $\mathrm{mM}$ glucose solution. After production, $50 \mu \mathrm{L}$ of $\mathrm{GUV}$ solution was collected from the bottom of the rotating chamber and deposited on a custom-built observation chamber. Separately, a buffered solution ( $80 \mathrm{mM}$ Tris $\mathrm{pH}$ 7.4 and $240 \mathrm{mM}$ glucose) was mixed with a $4 \mathrm{mg} \mathrm{mL}^{-1} 4 \mathrm{kDa}$ polyisocyanide hydrogel solution ${ }^{40}$ in a $1: 1$ volume ratio, and $50 \mu \mathrm{L}$ of the resulting solution was quickly added to the GUVs. The hydrogel was used to immobilize the GUVs, facilitating extended time-lapse imaging. After a few minutes, $2 \mu \mathrm{L}$ of 12 $\mu \mathrm{M}$ alpha-hemolysin solution (100 mM Tris- $\mathrm{HCl} \mathrm{pH}$ 7.5, $1 \mathrm{M}$ sodium chloride $(\mathrm{NaCl}), 7.5 \mathrm{mM}$ desthiobiotin (DTB)) was added to the observation chamber. Fluorescence intensity was analyzed manually using ImageJ and results plotted with MATLAB. Alpha-hemolysin was purified in-house according to Stranges et al. ${ }^{78}$

G-Actin Encapsulation. DOPC and ATTO 655 DOPE were mixed in a 99.9:0.1 molar ratio to prepare the lipid-in-oil dispersion. $100 \mu \mathrm{L}$ of $\mathrm{G}$-actin $(4.4 \mu \mathrm{M}, 9 \%$ labeled with Alexa Fluor 488) in G-buffer ( $5 \mathrm{mM}$ Tris- $\mathrm{HCl} \mathrm{pH} \mathrm{7.8,0.1} \mathrm{mM}$ 
$\mathrm{CaCl}_{2}, 0.02 \mathrm{mM}$ ATP and $4 \mathrm{mM} \mathrm{DTT}$ ) and $18.5 \% \mathrm{v} / \mathrm{v}$ OptiPrep was encapsulated in every experiment, only varying rotation speed and capillary size. For a capillary size of $25 \mu \mathrm{m}$, the flow rate was lowered to $2.5 \mu \mathrm{L} \mathrm{min}{ }^{-1}$ to reduce the pressure in the capillary setup. The encapsulated volume was reduced to $50 \mu \mathrm{L}$ in these experiments. GUVs were produced in an outer aqueous solution containing approximately $85 \mathrm{mM}$ glucose in Milli-Q water. G-actin was purchased from Hypermol and Alexa Fluor 488-labeled G-actin was obtained from Invitrogen. All proteins were handled according to instructions provided by the manufacturer. GUVs were imaged in the outer aqueous solution using confocal microscopy, 50 $\mu \mathrm{L}$ of GUV solution was deposited on a custom-made glass coverslip and covered. Microscopy was performed using a Nikon A1R-MP confocal microscope, using a Plan APO IR $60 \times$ water immersion objective. The $561 \mathrm{~nm}$ (laser power 1.0) and $488 \mathrm{~nm}$ (laser power 1.0) laser lines were used in combination with the appropriate emission filters to image the ATTO 655-labeled DOPE membrane and Alexa Fluor 488labeled G-actin, respectively.

Data Analysis of GUV Images. GUV size and inner intensity (Figure 1d, Figure 3a,c, and Figure S3) were obtained from $\mathrm{Z}$-stack images that were processed using custom-written Python software. The software performs feature tracking in each frame in three consecutive steps. First, the Canny edge detection algorithm ${ }^{79}$ is applied, then filling of the detected edges is achieved by applying the binary hole filling function from the ndimage module of the SciPy package, ${ }^{80}$ and next these features in each frame are located using the measure module of the scikit-image package ${ }^{81}$ for Python. The located features are linked together in a final step to group points belonging to the same GUV along the frame-axis. The radius of the GUVs was determined from the frame where the detected feature was largest and the inner intensity was also obtained from that respective frame and feature. User-based filtering was applied afterward to discard multilamellar structures, aggregates or similar. The software is available on GitHub (https:// github.com/GanzingerLab). The intensity was normalized to the mean of the distribution in Figure 3a.

PURE System Encapsulation. The codon-optimized construct encoding for meYFPco-LL-spinach (enhanced yellow fluorescent protein) described in Van Nies et al. ${ }^{82}$ was used. The sequence is codon-optimized for expression in the PURE system, and the template includes the $\mathrm{T} 7$ promoter and terminator. A linear DNA template was employed to observe fluorescence readout of the level of synthesized protein. The linear DNA construct was obtained by polymerase chain reaction (forward primer: GCGAAATTAATACGACTCACTATAGGGAGACC, reverse primer: AAAAAACCCCTCAAGACCCGTTTAGAGG). Amplification products were checked on a $1 \%$ agarose gel and were purified using the Wizard PCR cleanup kit (Promega). DNA concentration and purity were measured using a ND-1000 UV-vis Spectrophotometer (Nanodrop Technologies).

The full sequence of the meYFPco-LL-spinach linear construct is as follows:

5' - GCGAAATTAATACGACTCACTATAGGGAGACCACAACGGTTTCCCTCTAGAAATAATTTTGTTTAACTTTAAGAAGGAGATATACATATGCGGGGTTCTCATCATCATCATCATCATGGTATGGCTAGCATGACTGGTGGACAGCAAA T GGG T C G G GA T C T GTA C GA C GATGACGATAAGGATCCGATGGTTAGCAAAGGCGAAGAAC T G T T T A C G G G C G T G G T G C C GA T T C T G -
GTGGAACTGGACGGCGACGTGAACGGTCACAAA T T CAGCGT T T C GGG C GAAGGTGAAGG CGAT GCGACCTAT GGTAAACT GAC GC T GAAATTTATTTGCACCACCGGTAAACTGCCGGTGCCGT GGCCGACCCTGGTTACCACGT T T G GT T A T G G C C T G CA G T G T T T C G C G C G C T A C C C G G ATCATATGAAACAACACGACTTTTTCAAATCTGCCATGCCGGAAGGTTATGTGCAGGAACGTACGATTTTCTTTAAAGATGACGGCAACTACAAAACCCGCGCAGAAGTCAAATTTGAAGGTGATACGCTGGTGAACCGTATTGAACTGAAAGGCATCGATTTCAAAGAAGACGGTAATATCCTGGGCCATAAACTGGAATACAACTACAACTCCCACAACGTTTACATCATGGCAGATAAACAGAAAAACGGTATCAAAGTCAACTTCAAAATCCGCCATAACATCGAAGATGGCTCAGTGCAACTGGCTGACCACTACCAGCAAAA C A C C C C GA T C G G T GA T G G C C C G G T T C TGCTGCCGGACAATCATTATCTGAGCTACCAGTCTAAACTGAGTAAAGATCCGAACGAAAAACGTGACCACAT G G T C C T G C T G GAA T T T G T GA C G G C G G C T G GTATTACGCTGGGCATGGATGAACTGTATAAATGAAAGCTTCCCGGGAAAGTATATATGAGTAAAGATATCGACGCAACTGAATGAAATGGTGAAGGACGGGTCCAGGT G T G G C T G C T T C G G CA G T G CA G C T T G T T GAGTAGAGTGTGAGCTCCGTAACTAGTCGCGT CGATAT C C C CGGGCTAGCATAAC C C C T T GG GGCCTCTAAACGGGTCTTGAGGGGTTTTTT-3’.

DOPC and rhodamine-PE were used in a 99.9:0.1 molar ratio for the lipid-in-oil dispersion, $0.01 \mathrm{~mol} \%$ of 18:0 PEG2000 PE was used when explicitly mentioned. PUREfrex2.0 (GeneFrontier Corporation, Japan) was used following storage and handling instructions provided by the supplier. Linear DNA template was added at a concentration of $5 \mathrm{nM}$. Reactions of $40 \mu \mathrm{L}$ were assembled in test tubes and supplemented with $5 \% \mathrm{v} / \mathrm{v}$ OptiPrep (higher ratios negatively interfered with the PURE reaction) and kept on ice. GUVs were produced in an outer aqueous solution composed of 220 $\mathrm{mM}$ glucose in Milli-Q water. The flow rate was kept at $2.5 \mu \mathrm{L}$ $\min ^{-1}$ for $8 \mathrm{~min}$ in total, given the limited availability of inner aqueous solution. After production, $25 \mu \mathrm{L}$ of GUV solution was transferred to the observation chamber, together with 25 $\mu \mathrm{L}$ of additional outer aqueous solution composed of $35 \mathrm{mM}$ glucose and $50 \% \mathrm{v} / \mathrm{v}$ PURE buffer. YFP expression was monitored at $37{ }^{\circ} \mathrm{C}$ by confocal imaging using a Nikon A1R Laser scanning confocal microscope equipped with an SR Apo TIRF $100 \times$ oil-immersion objective. The $561 \mathrm{~nm}$ (laser power 5.0) and $488 \mathrm{~nm}$ (laser power 20.0) laser lines were used in combination with the appropriate emission filters to image the rhodamine-PE membrane and YFP, respectively. The software NIS (Nikon) was used for image acquisition and the settings were identical for all experiments. Samples were mounted on a temperature-controlled stage maintained at $37{ }^{\circ} \mathrm{C}$ during imaging up to $5 \mathrm{~h}$.

Image analysis was carried out in MATLAB version R2020b using the script published by Blanken et al. ${ }^{83}$ Briefly, the script reads the split-channel tiff files, identifies the GUVs, indexes them, and then stores the indexed variables in the data file. The script uses a sharpening filter on the rhodamine-PE image, the GUV lumen is determined by a flood filling step followed by a binarization phase with a cutoff of 200 . An erosion step was conducted to filter segments relative to lipid aggregates and other sources of noise. Any segments with a circularity of less than 0.5 or greater than 2 have been excluded. For each GUV, average rhodamine-PE intensity, average YFP intensity and 
YFP intensity variance were determined. The box plots of the YFP intensity in the lumen were also generated in MATLAB version $\mathrm{R} 2020 \mathrm{~b}$.

Actin Cortex. GUVs were prepared using a mixture of DOPC and DGS-NTA(Ni) lipids in a 50:1 molar ratio. Gactin $(4.4 \mu \mathrm{M}, 9 \%$ labeled with Alexa Fluor 647$)$, profilin (3.3 $\mu \mathrm{M}), \operatorname{Arp} 2 / 3(100 \mathrm{nM})$, and VCA $(0.6 \mu \mathrm{M})$ were added to a solution containing F-buffer $(20 \mathrm{mM}$ Tris- $\mathrm{HCl}$ pH 7.4, $50 \mathrm{mM}$ $\mathrm{KCl}, 2 \mathrm{mM} \mathrm{MgCl}_{2}, 0.5 \mathrm{mM}$ ATP and $1 \mathrm{mM} \mathrm{DTT}$ ) and $18.5 \%$ v/v OptiPrep. To minimize photobleaching, an oxygenscavenger system ${ }^{84}(1 \mathrm{mM}$ protocatechuic acid (PCA) and $50 \mathrm{nM}$ protocatechuate-3,4-dioxygenase (PCD)) was also added to the solution. GUVs were produced in an outer aqueous solution containing $200 \mathrm{mM}$ glucose in Milli-Q water. After production, $25 \mu \mathrm{L}$ of GUV solution was collected from the bottom of the rotating chamber and deposited on a custom-built observation chamber, to which an additional 25 $\mu \mathrm{L}$ of a buffered solution ( $40 \mathrm{mM}$ Tris $\mathrm{pH} 7.4$ and $125 \mathrm{mM}$ glucose) was added. Unless specified otherwise, all chemicals were purchased from Sigma-Aldrich. All proteins, except VCA, which was purified in-house, ${ }^{85}$ were purchased from Hypermol and dissolved according to instructions provided by the manufacturer. G-actin was dialyzed in G-buffer ( $5 \mathrm{mM}$ Tris$\mathrm{HCl} \mathrm{pH} 7.8$ and $0.1 \mathrm{mM} \mathrm{CaCl}_{2}$ ) before storage at $-80{ }^{\circ} \mathrm{C}$.

DNA Origami Nanostructures Encapsulation. The DNA origami design was adapted from Jeon et al. ${ }^{63}$ by removing the 3 ' sequence ("sticky ends") mediating multimerization, thus keeping them monomeric. An additional $12 \mathrm{nt}$ sequence was added at the $5^{\prime}$ end to allow binding to the membrane via a cholesterol-oligonucleotide anchor. Nanostructures were folded by thermal annealing (from 95 to $23^{\circ} \mathrm{C}$, $-0.5{ }^{\circ} \mathrm{C} \mathrm{min}^{-1}$ ) and used at $1 \mu \mathrm{M}$ in buffered solution (50 $\mathrm{mM}$ Tris $\mathrm{pH}$ 7.0, $2 \mathrm{mM} \mathrm{MgCl}_{2}$, and $200 \mathrm{mM}$ sucrose). Right before encapsulation, $2 \mu \mathrm{M}$ of cholesterol-oligonucleotides were added to this buffer. As an outer aqueous phase, $50 \mathrm{mM}$ Tris $\mathrm{pH} 7.0,2 \mathrm{mM} \mathrm{MgCl}_{2}$ and $200 \mathrm{mM}$ glucose was used. Experiments were performed using PEEK capillary tubing.

SUV Encapsulation. SUVs were prepared using DOPC and ATTO 488 DOPE in a 99:1 molar ratio. Under gentle nitrogen flow, chloroform was evaporated to obtain a homogeneous lipid film. The lipid film was then desiccated for a minimum of $3 \mathrm{~h}$ to remove any remaining solvent traces, after which it was rehydrated in phosphate-buffered saline buffer (PBS buffer) at $4 \mathrm{mg} \mathrm{mL}^{-1}$ by vortexing. Afterward the solution was sonicated in aliquots of $20 \mu \mathrm{L}$ for $2 \times 30 \mathrm{~min}$. It was then diluted to $0.5 \mathrm{mg} \mathrm{mL}^{-1}$ for further use. DOPC and ATTO 655 DOPE were used in a 99.9:1 molar ratio for the lipid-in-oil dispersion. For encapsulation, the SUVs were diluted $10 \times$ in PBS buffer and $18.5 \% \mathrm{v} / \mathrm{v}$ OptiPrep was added. The outer aqueous phase consisted of $313 \mathrm{mM}$ glucose in Milli-Q water.

Bacteria Encapsulation. DOPC, 18:1 Liss Rhod PE, and 18:0 PEG2000 PE were used in a 98.9:0.1:1 molar ratio for the lipid-in-oil dispersion. A saturated lysogeny broth (LB) culture of Escherichia coli expressing green fluorescent protein (GFP$\mathrm{HU}$ ) was centrifuged and the pellet resuspended in a buffered solution $(50 \mathrm{mM}$ Tris $\mathrm{pH} 7.5,5 \mathrm{mM} \mathrm{NaCl}$ and $200 \mathrm{mM}$ sucrose) and used for encapsulation. As an outer aqueous phase, $50 \mathrm{mM}$ Tris $\mathrm{pH} 7.5,5 \mathrm{mM} \mathrm{NaCl}$ and $200 \mathrm{mM}$ glucose was used. Experiments were performed using PEEK capillary tubing.

\section{ASSOCIATED CONTENT}

\section{Supporting Information}

The Supporting Information is available free of charge at https://pubs.acs.org/doi/10.1021/acssynbio.1c00068.

Influence of environmental conditions on lipid adsorption kinetics; UV-vis absorbance of different lipid-in-oil mixtures; size distributions of GUVs for different capillary sizes and rotation speeds; representative fields of view of GUVs encapsulating G-actin, the PURE system, a minimal actin cortex, DNA origami nanostructures, SUVs, and GFP-HU expressing E. coli bacteria; schematic of a single DNA nanostructure (PDF)

Time-lapse movie of encapsulated GFP-HU expressing E. coli bacteria (MP4)

\section{AUTHOR INFORMATION}

\section{Corresponding Authors}

Gijsje H. Koenderink - Department of Bionanoscience, Kavli Institute of Nanoscience Delft, Delft University of Technology, 2629 HZ Delft, The Netherlands; 1 orcid.org/0000-00027823-8807; Email: g.h.koenderink@tudelft.nl

Kristina A. Ganzinger - Department of Living Matter, AMOLF, 1098 XG Amsterdam, The Netherlands; ○ orcid.org/0000-0001-9106-9406; Email: k.ganzinger@ amolf.nl

\section{Authors}

Lori Van de Cauter - Department of Living Matter, AMOLF, 1098 XG Amsterdam, The Netherlands; 10 orcid.org/00000002-3459-8464

Federico Fanalista - Department of Bionanoscience, Kavli Institute of Nanoscience Delft, Delft University of Technology, 2629 HZ Delft, The Netherlands

Lennard van Buren - Department of Bionanoscience, Kavli Institute of Nanoscience Delft, Delft University of Technology, 2629 HZ Delft, The Netherlands

Nicola De Franceschi - Department of Bionanoscience, Kavli Institute of Nanoscience Delft, Delft University of Technology, 2629 HZ Delft, The Netherlands; 이 orcid.org/0000-00027221-613X

Elisa Godino - Department of Bionanoscience, Kavli Institute of Nanoscience Delft, Delft University of Technology, 2629 HZ Delft, The Netherlands

Sharon Bouw - Department of Living Matter, AMOLF, 1098 XG Amsterdam, The Netherlands

Christophe Danelon - Department of Bionanoscience, Kavli Institute of Nanoscience Delft, Delft University of Technology, 2629 HZ Delft, The Netherlands; 이이. orid.org/0000-00020961-6640

Cees Dekker - Department of Bionanoscience, Kavli Institute of Nanoscience Delft, Delft University of Technology, 2629 HZ Delft, The Netherlands; 10 orcid.org/0000-0001-6273$071 \mathrm{X}$

Complete contact information is available at:

https://pubs.acs.org/10.1021/acssynbio.1c00068

\section{Author Contributions}

K.A.G. and G.H.K. designed and directed the project; L.V.d.C., F.F., L.v.B., N.D.F., E.G., and S.B. performed the experiments; L.V.d.C., F.F., and L.v.B. drafted the manuscript and designed the figures; all authors provided critical feedback and helped shape the research and manuscript. 


\section{Author Contributions}

${ }^{\S}$ L.V.d.C., F.F., and L.v.B. contributed equally.

\section{Notes}

The authors declare no competing financial interest.

\section{ACKNOWLEDGMENTS}

We thank Paul Kouwer (Radboud University) for the kind gift of the polyisocyanide gel, and Josef Melcr and Siewert-Jan Marrink for useful discussions. We acknowledge financial support by the "BaSyC - Building a Synthetic Cell" Gravitation grant (024.003.019) of The Netherlands Ministry of Education, Culture and Science (OCW) and The Netherlands Organization for Scientific Research (NWO) (G.H.K., C. Dekker, and C. Danelon) and NWO-WISE funding (K.A.G.).

\section{REFERENCES}

(1) Ganzinger, K. A., and Schwille, P. (2019) More from Less Bottom-up Reconstitution of Cell Biology. J. Cell Sci. 132, 11.

(2) Göpfrich, K., Platzman, I., and Spatz, J. P. (2018) Mastering Complexity: Towards Bottom-up Construction of Multifunctional Eukaryotic Synthetic Cells. Trends Biotechnol. 36 (9), 938-951.

(3) Lagny, T. J., and Bassereau, P. (2015) Bioinspired MembraneBased Systems for a Physical Approach of Cell Organization and Dynamics: Usefulness and Limitations. Interface Focus 5, 20150038.

(4) BaSyC - Building a Synthetic Cell. https://www.basyc.nl/ (accessed Nov 23, 2020).

(5) Synthetic Cell Initiative. https://www.syntheticcell.eu/ (accessed Nov 23, 2020).

(6) Build-a-Cell. https://www.buildacell.org (accessed Nov 23, 2020).

(7) Schwille, P., Spatz, J., Landfester, K., Bodenschatz, E., Herminghaus, S., Sourjik, V., Erb, T. J., Bastiaens, P., Lipowsky, R., Hyman, A., Dabrock, P., Baret, J.-C., Vidakovic-Koch, T., Bieling, P., Dimova, R., Mutschler, H., Robinson, T., Tang, T.-Y. D., Wegner, S., and Sundmacher, K. (2018) MaxSynBio: Avenues Towards Creating Cells from the Bottom Up. Angew. Chem., Int. Ed. 57 (41), 1338213392.

(8) Walde, P., Cosentino, K., Engel, H., and Stano, P. (2010) Giant Vesicles: Preparations and Applications. ChemBioChem 11 (7), 848865.

(9) Dimova, R., and Marques, C. M. (2019) The Giant Vesicle Book, 1st ed., CRC Press.

(10) Dimova, R. (2019) Giant Vesicles and Their Use in Assays for Assessing Membrane Phase State, Curvature, Mechanics, and Electrical Properties. Annu. Rev. Biophys. 48 (1), 93-119.

(11) Papahadjopoulos, D., and Kimelberg, H. K. (1974) Phospholipid Vesicles (Liposomes) as Models for Biological Membranes: Their Properties and Interactions with Cholesterol and Proteins. Prog. Surf. Sci. 4 (C), 141-144. IN9, 145-232.

(12) Reeves, J. P., and Dowben, R. M. (1969) Formation and Properties of Thin-Walled Phospholipid Vesicles. J. Cell. Physiol. 73 (1), 49-60.

(13) Angelova, M. I., and Dimitrov, D. S. (1986) Liposome Electroformation. Faraday Discuss. Chem. Soc. 81, 303-311.

(14) Montes, L.-R., Alonso, A., Goñi, F. M., and Bagatolli, L. A. (2007) Giant Unilamellar Vesicles Electroformed from Native Membranes and Organic Lipid Mixtures under Physiological Conditions. Biophys. J. 93 (10), 3548-3554.

(15) Breton, M., Amirkavei, M., and Mir, L. M. (2015) Optimization of the Electroformation of Giant Unilamellar Vesicles (GUVs) with Unsaturated Phospholipids. J. Membr. Biol. 248 (5), 827-835.

(16) Dimova, R., Aranda, S., Bezlyepkina, N., Nikolov, V., Riske, K. A., and Lipowsky, R. (2006) A Practical Guide to Giant Vesicles. Probing the Membrane Nanoregime via Optical Microscopy. J. Phys.: Condens. Matter 18 (28), S1151-S1176.
(17) Horger, K. S., Estes, D. J., Capone, R., and Mayer, M. (2009) Films of Agarose Enable Rapid Formation of Giant Liposomes in Solutions of Physiologic Ionic Strength. J. Am. Chem. Soc. 131 (5), $1810-1819$.

(18) Weinberger, A., Tsai, F.-C., Koenderink, G. H., Schmidt, T. F., Itri, R., Meier, W., Schmatko, T., Schröder, A., and Marques, C. (2013) Gel-Assisted Formation of Giant Unilamellar Vesicles. Biophys. J. 105 (1), 154-164.

(19) López Mora, N., Hansen, J. S., Gao, Y., Ronald, A. A., Kieltyka, R., Malmstadt, N., and Kros, A. (2014) Preparation of Size Tunable Giant Vesicles from Cross-Linked Dextran(Ethylene Glycol) Hydrogels. Chem. Commun. 50 (16), 1953-1955.

(20) Schultze, J., Vagias, A., Ye, L., Prantl, E., Breising, V., Best, A., Koynov, K., Marques, C. M., and Butt, H.-J. (2019) Preparation of Monodisperse Giant Unilamellar Anchored Vesicles Using Micropatterned Hydrogel Substrates. ACS Omega 4 (5), 9393-9399.

(21) Hu, P. C., Li, S., and Malmstadt, N. (2011) Microfluidic Fabrication of Asymmetric Giant Lipid Vesicles. ACS Appl. Mater. Interfaces 3 (5), 1434-1440.

(22) Ito, H., Yamanaka, T., Kato, S., Hamada, T., Takagi, M., Ichikawa, M., and Yoshikawa, K. (2013) Dynamical Formation of Lipid Bilayer Vesicles from Lipid-Coated Droplets across a Planar Monolayer at an Oil/Water Interface. Soft Matter 9 (40), 9539-9547.

(23) van Swaay, D., and deMello, A. (2013) Microfluidic Methods for Forming Liposomes. Lab Chip 13 (5), 752.

(24) Funakoshi, K., Suzuki, H., and Takeuchi, S. (2007) Formation of Giant Lipid Vesiclelike Compartments from a Planar Lipid Membrane by a Pulsed Jet Flow. J. Am. Chem. Soc. 129 (42), 1260812609.

(25) Stachowiak, J. C., Richmond, D. L., Li, T. H., Liu, A. P., Parekh, S. H., and Fletcher, D. A. (2008) Unilamellar Vesicle Formation and Encapsulation by Microfluidic Jetting. Proc. Natl. Acad. Sci. U. S. A. 105 (12), 4697-4702.

(26) Richmond, D. L., Schmid, E. M., Martens, S., Stachowiak, J. C., Liska, N., and Fletcher, D. A. (2011) Forming Giant Vesicles with Controlled Membrane Composition, Asymmetry, and Contents. Proc. Natl. Acad. Sci. U. S. A. 108 (23), 9431-9436.

(27) Deshpande, S., Caspi, Y., Meijering, A. E. C., and Dekker, C. (2016) Octanol-Assisted Liposome Assembly on Chip. Nat. Commun. 7 (1), 10447.

(28) Abkarian, M., Loiseau, E., and Massiera, G. (2011) Continuous Droplet Interface Crossing Encapsulation (CDICE) for High Throughput Monodisperse Vesicle Design. Soft Matter 7 (10), 4610-4614.

(29) Blosser, M. C., Horst, B. G., and Keller, S. L. (2016) CDICE Method Produces Giant Lipid Vesicles under Physiological Conditions of Charged Lipids and Ionic Solutions. Soft Matter 12 (35), 7364-7371.

(30) Deek, J., Maan, R., Loiseau, E., and Bausch, A. R. (2018) Reconstitution of Composite Actin and Keratin Networks in Vesicles. Soft Matter 14 (10), 1897-1902.

(31) Keber, F. C., Loiseau, E., Sanchez, T., DeCamp, S. J., Giomi, L., Bowick, M. J., Marchetti, M. C., Dogic, Z., and Bausch, A. R. (2014) Topology and Dynamics of Active Nematic Vesicles. Science 345 (6201), 1135-1139.

(32) Litschel, T., Ganzinger, K. A., Movinkel, T., Heymann, M., Robinson, T., Mutschler, H., and Schwille, P. (2018) Freeze-Thaw Cycles Induce Content Exchange between Cell-Sized Lipid Vesicles. New J. Phys. 20, 055008.

(33) Litschel, T., Ramm, B., Maas, R., Heymann, M., and Schwille, P. (2018) Beating Vesicles: Encapsulated Protein Oscillations Cause Dynamic Membrane Deformations. Angew. Chem., Int. Ed. 57 (50), 16286-16290.

(34) Berry, J. D., Neeson, M. J., Dagastine, R. R., Chan, D. Y. C., and Tabor, R. F. (2015) Measurement of Surface and Interfacial Tension Using Pendant Drop Tensiometry. J. Colloid Interface Sci. 454, 226237. 
(35) KNMI - Daggegevens van het weer in Nederland. http:// projects.knmi.nl/klimatologie/daggegevens/selectie.cgi (accessed Dec $4,2020)$.

(36) Loiseau, E., Schneider, J. A. M., Keber, F. C., Pelzl, C., Massiera, G., Salbreux, G., and Bausch, A. R. (2016) Shape Remodeling and Blebbing of Active Cytoskeletal Vesicles. Sci. Adv. 2 (4), No. e1500465.

(37) Dürre, K., and Bausch, A. R. (2019) Formation of Phase Separated Vesicles by Double Layer CDICE. Soft Matter 15 (47), 9676-9681.

(38) Song, L., Hobaugh, M. R., Shustak, C., Cheley, S., Bayley, H., and Gouaux, J. E. (1996) Structure of Staphylococcal A-Hemolysin, a Heptameric Transmembrane Pore. Science 274 (5294), 1859-1866.

(39) Ostolaza, H., Bartolomé, B., Ortiz de Zárate, I., de la Cruz, F., and Goñi, F. M. (1993) Release of Lipid Vesicle Contents by the Bacterial Protein Toxin A-Haemolysin. Biochim. Biophys. Acta, Biomembr. 1147 (1), 81-88.

(40) Kouwer, P. H. J., Koepf, M., Le Sage, V. A. A., Jaspers, M., van Buul, A. M., Eksteen-Akeroyd, Z. H., Woltinge, T., Schwartz, E., Kitto, H. J., Hoogenboom, R., Picken, S. J., Nolte, R. J. M., Mendes, E., and Rowan, A. E. (2013) Responsive Biomimetic Networks from Polyisocyanopeptide Hydrogels. Nature 493 (7434), 651-655.

(41) Lira, R. B., Steinkühler, J., Knorr, R. L., Dimova, R., and Riske, K. A. (2016) Posing for a Picture: Vesicle Immobilization in Agarose Gel. Sci. Rep. 6 (1), 25254.

(42) Mulla, Y., Aufderhorst-Roberts, A., and Koenderink, G. H. (2018) Shaping up Synthetic Cells. Phys. Biol. 15 (4), 041001.

(43) Claudet, C., In, M., and Massiera, G. (2016) Method to Disperse Lipids as Aggregates in Oil for Bilayers Production. Eur. Phys. J. E: Soft Matter Biol. Phys. 39 (1), 9.

(44) Doerr, A., de Reus, E., van Nies, P., van der Haar, M., Wei, K., Kattan, J., Wahl, A., and Danelon, C. (2019) Modelling Cell-Free RNA and Protein Synthesis with Minimal Systems. Phys. Biol. 16 (2), 025001 .

(45) Saito, H., Kato, Y., Le Berre, M., Yamada, A., Inoue, T., Yosikawa, K., and Baigl, D. (2009) Time-Resolved Tracking of a Minimum Gene Expression System Reconstituted in Giant Liposomes. ChemBioChem 10 (10), 1640-1643.

(46) Sunami, T., Sato, K., Matsuura, T., Tsukada, K., Urabe, I., and Yomo, T. (2006) Femtoliter Compartment in Liposomes for in Vitro Selection of Proteins. Anal. Biochem. 357 (1), 128-136.

(47) Hosoda, K., Sunami, T., Kazuta, Y., Matsuura, T., Suzuki, H., and Yomo, T. (2008) Quantitative Study of the Structure of Multilamellar Giant Liposomes As a Container of Protein Synthesis Reaction. Langmuir 24 (23), 13540-13548.

(48) Ota, S., Yoshizawa, S., and Takeuchi, S. (2009) Microfluidic Formation of Monodisperse, Cell-Sized, and Unilamellar Vesicles. Angew. Chem., Int. Ed. 48 (35), 6533-6537.

(49) Shimizu, Y., Inoue, A., Tomari, Y., Suzuki, T., Yokogawa, T., Nishikawa, K., and Ueda, T. (2001) Cell-Free Translation Reconstituted with Purified Components. Nat. Biotechnol. 19 (8), $751-755$.

(50) Tsai, F.-C., Stuhrmann, B., and Koenderink, G. H. (2011) Encapsulation of Active Cytoskeletal Protein Networks in Cell-Sized Liposomes. Langmuir 27 (16), 10061-10071.

(51) Castile, J. D., and Taylor, K. M. G. (1999) Factors Affecting the Size Distribution of Liposomes Produced by Freeze-Thaw Extrusion. Int. J. Pharm. 188 (1), 87-95.

(52) Yamashita, Y., Oka, M., Tanaka, T., and Yamazaki, M. (2002) A New Method for the Preparation of Giant Liposomes in High Salt Concentrations and Growth of Protein Microcrystals in Them. Biochim. Biophys. Acta, Biomembr. 1561 (2), 129-134.

(53) Cheffings, T. H., Burroughs, N. J., and Balasubramanian, M. K. (2016) Actomyosin Ring Formation and Tension Generation in Eukaryotic Cytokinesis. Curr. Biol. 26 (15), R719-R737.

(54) Koenderink, G. H., and Paluch, E. K. (2018) Architecture Shapes Contractility in Actomyosin Networks. Curr. Opin. Cell Biol. $50,79-85$.
(55) Machesky, L. M., and Gould, K. L. (1999) The Arp2/3 Complex: A Multifunctional Actin Organizer. Curr. Opin. Cell Biol. 11 (1), 117-121.

(56) Pantaloni, D., Boujemaa, R., Didry, D., Gounon, P., and Carlier, M.-F. (2000) The Arp2/3 Complex Branches Filament Barbed Ends: Functional Antagonism with Capping Proteins. Nat. Cell Biol. 2 (7), 385-391.

(57) Veltman, D. M., and Insall, R. H. (2010) WASP Family Proteins: Their Evolution and Its Physiological Implications. Mol. Biol. Cell 21 (16), 2880-2893.

(58) Sonal Ganzinger, K. A., Vogel, S. K., Mücksch, J., Blumhardt, P., and Schwille, P. (2019) Myosin-II Activity Generates a Dynamic Steady State with Continuous Actin Turnover in a Minimal Actin Cortex. J. Cell Sci. 132 (4), No. jcs219899.

(59) Wang, R., and Carlsson, A. E. (2015) How Capping Protein Enhances Actin Filament Growth and Nucleation on Biomimetic Beads. Phys. Biol. 12 (6), 066008.

(60) Akin, O., and Mullins, R. D. (2008) Capping Protein Increases the Rate of Actin-Based Motility by Promoting Filament Nucleation by the Arp2/3 Complex. Cell 133, 841-851.

(61) Guevorkian, K., Manzi, J., Pontani, L.-L., Brochard-Wyart, F., and Sykes, C. (2015) Mechanics of Biomimetic Liposomes Encapsulating an Actin Shell. Biophys. J. 109 (12), 2471-2479.

(62) Pontani, L.-L., van der Gucht, J., Salbreux, G., Heuvingh, J., Joanny, J.-F., and Sykes, C. (2009) Reconstitution of an Actin Cortex Inside a Liposome. Biophys. J. 96 (1), 192-198.

(63) Jeon, B., Nguyen, D. T., Abraham, G. R., Conrad, N., Fygenson, D. K., and Saleh, O. A. (2018) Salt-Dependent Properties of a Coacervate-like, Self-Assembled DNA Liquid. Soft Matter 14 (34), 7009-7015.

(64) Bolinger, P.-Y., Stamou, D., and Vogel, H. (2008) An Integrated Self-Assembled Nanofluidic System for Controlled Biological Chemistries. Angew. Chem., Int. Ed. 47 (30), 5544-5549.

(65) Lee, K. Y., Park, S.-J., Lee, K. A., Kim, S.-H., Kim, H., Meroz, Y., Mahadevan, L., Jung, K.-H., Ahn, T. K., Parker, K. K., and Shin, K. (2018) Photosynthetic Artificial Organelles Sustain and Control ATPDependent Reactions in a Protocellular System. Nat. Biotechnol. 36 (6), 530-535.

(66) Hindley, J. W., Elani, Y., McGilvery, C. M., Ali, S., Bevan, C. L., Law, R. V., and Ces, O. (2018) Light-Triggered Enzymatic Reactions in Nested Vesicle Reactors. Nat. Commun. 9 (1), 1093.

(67) Phillips, R., Kondev, J., and Theriot, J. (2008) Physical Biology of the Cell, Garland Science, Taylor \& Francis Group, New York.

(68) Miller, T. E., Beneyton, T., Schwander, T., Diehl, C., Girault, M., McLean, R., Chotel, T., Claus, P., Cortina, N. S., Baret, J.-C., and Erb, T. J. (2020) Light-Powered $\mathrm{CO}_{2}$ Fixation in a Chloroplast Mimic with Natural and Synthetic Parts. Science 368 (6491), 649654

(69) Lehtinen, O.-P., Nugroho, R. W. N., Lehtimaa, T., Vierros, S., Hiekkataipale, P., Ruokolainen, J., Sammalkorpi, M., and Österberg, M. (2017) Effect of Temperature, Water Content and Free Fatty Acid on Reverse Micelle Formation of Phospholipids in Vegetable Oil. Colloids Surf., B 160, 355-363.

(70) Koynova, R., and Tenchov, B. (2013) Phase Transitions and Phase Behavior of Lipids. In Encyclopedia of Biophysics (Roberts, G. C. K., Ed.) pp 1841-1854, Springer Berlin Heidelberg: Berlin, Heidelberg. DOI: 10.1007/978-3-642-16712-6 542.

(71) Karamdad, K., Law, R. V., Seddon, J. M., Brooks, N. J., and Ces, O. (2015) Preparation and Mechanical Characterisation of Giant Unilamellar Vesicles by a Microfluidic Method. Lab Chip 15 (2), $557-562$.

(72) Moga, A., Yandrapalli, N., Dimova, R., and Robinson, T. (2019) Optimization of the Inverted Emulsion Method for HighYield Production of Biomimetic Giant Unilamellar Vesicles. ChemBioChem 20 (20), 2674-2682.

(73) Schaich, M., Sobota, D., Sleath, H., Cama, J., and Keyser, U. F. (2020) Characterization of Lipid Composition and Diffusivity in OLA Generated Vesicles. Biochim. Biophys. Acta, Biomembr. 1862 (9), 183359. 
(74) Garenne, D., Libchaber, A., and Noireaux, V. (2020) Membrane Molecular Crowding Enhances MreB Polymerization to Shape Synthetic Cells from Spheres to Rods. Proc. Natl. Acad. Sci. U. S. A. 117 (4), 1902-1909.

(75) Raleigh, Lord (1879) VI. On the Capillary Phenomena of Jets.

Proc. R. Soc. London 29, 71-79.

(76) Bashirzadeh, Y., Redford, S. A., Lorpaiboon, C., Groaz, A., Litschel, T., Schwille, P., Hocky, G. M., Dinner, A. R., and Liu, A. P. (2020) Actin Crosslinker Competition and Sorting Drive Emergent GUV Size-Dependent Actin Network Architecture. bioRxiv, Apr 21, 2020, 2020.10.03.322354. DOI: 10.1101/2020.10.03.322354.

(77) Tsai, F.-C., and Koenderink, G. H. (2015) Shape Control of Lipid Bilayer Membranes by Confined Actin Bundles. Soft Matter 11 (45), 8834-8847.

(78) Stranges, P. B., Palla, M., Kalachikov, S., Nivala, J., Dorwart, M., Trans, A., Kumar, S., Porel, M., Chien, M., Tao, C., Morozova, I., Li, Z., Shi, S., Aberra, A., Arnold, C., Yang, A., Aguirre, A., Harada, E. T., Korenblum, D., Pollard, J., Bhat, A., Gremyachinskiy, D., Bibillo, A., Chen, R., Davis, R., Russo, J. J., Fuller, C. W., Roever, S., Ju, J., and Church, G. M. (2016) Design and Characterization of a NanoporeCoupled Polymerase for Single-Molecule DNA Sequencing by Synthesis on an Electrode Array. Proc. Natl. Acad. Sci. U. S. A. 113 (44), E6749-E6756.

(79) Canny, J. (1986) A Computational Approach to Edge Detection. IEEE Transactions on Pattern Analysis and Machine Intelligence PAMI-8 (6), 679-698.

(80) Virtanen, P., Gommers, R., Oliphant, T. E., Haberland, M., Reddy, T., Cournapeau, D., Burovski, E., Peterson, P., Weckesser, W., Bright, J., van der Walt, S. J., Brett, M., Wilson, J., Millman, K. J., Mayorov, N., Nelson, A. R. J., Jones, E., Kern, R., Larson, E., Carey, C. J., Polat, I., Feng, Y., Moore, E. W., VanderPlas, J., Laxalde, D., Perktold, J., Cimrman, R., Henriksen, I., Quintero, E. A., Harris, C. R., Archibald, A. M., Ribeiro, A. H., Pedregosa, F., and van Mulbregt, P. (2020) SciPy 1.0 Contributors. SciPy 1.0: Fundamental Algorithms for Scientific Computing in Python. Nat. Methods 17 (3), 261-272.

(81) van der Walt, S., Schönberger, J. L., Nunez-Iglesias, J., Boulogne, F., Warner, J. D., Yager, N., Gouillart, E., and Yu, T. (2014) Scikit-Image: Image Processing in Python. PeerJ 2, No. e453.

(82) van Nies, P., Canton, A. S., Nourian, Z., and Danelon, C. (2015) Monitoring MRNA and Protein Levels in Bulk and in Model Vesicle-Based Artificial Cells. Methods Enzymol. 550, 187-214.

(83) Blanken, D., van Nies, P., and Danelon, C. (2019) Quantitative Imaging of Gene-Expressing Liposomes Reveals Rare Favorable Phenotypes. Phys. Biol. 16 (4), 045002.

(84) Aitken, C. E., Marshall, R. A., and Puglisi, J. D. (2008) An Oxygen Scavenging System for Improvement of Dye Stability in Single-Molecule Fluorescence Experiments. Biophys. J. 94 (5), 18261835.

(85) Havrylenko, S., Noguera, P., Abou-Ghali, M., Manzi, J., Faqir, F., Lamora, A., Guérin, C., Blanchoin, L., and Plastino, J. (2015) WAVE Binds Ena/VASP for Enhanced Arp2/3 Complex-Based Actin Assembly. Mol. Biol. Cell 26 (1), 55-65. 